Keywords: atypical glandular cells of uncertain significance; borderline glandular cells; liquid-based cytology; HR HPV triage; intraepithelial neoplasia

\title{
Management of borderline change in endocervical cells: a more dependable approach
}

\author{
S Kodiathodi ${ }^{*}, 1$, S Chattopadhyay ${ }^{2}$, A Baldwin ${ }^{1}$ and P Franks ${ }^{1}$ \\ ${ }^{1}$ Department of Obstetrics and Gynaecology, Wansbeck Hospital, Woodhorn Lane, Ashington, Northumberland NE63 9JJ, UK \\ and ${ }^{2}$ Department of Gynaecological Oncology, St James's University Hospital, Beckett Street, Leeds, West Yorkshire LS9 7TF, UK
}

Background: There are limited data and guidance from the UK on borderline nuclear change in endocervical cells (BNCs). The objective of this study is to determine the clinical outcome of women with BNCs, to determine the accuracy of colposcopy and propose a more robust management algorithm.

Methods: This is a retrospective review of all BNC referrals between January 2006 and December 2011 at the Northumbria Healthcare Trust. Histological diagnosis was based on high-grade histology (CIN 2 or worse). Any high-grade histology in the first year of follow-up was included in the final diagnosis.

Results: Of the 9001 new referrals, 167 women had BNCs. Thirty-seven (22\%) were diagnosed with high-grade histology on initial assessment. Sixty women had satisfactory and negative colposcopy, out of which 7 (12\%) were detected with high-grade histology/cytology in the first year of follow-up. Overall, 50 high-grade histology (30\%), including two invasive carcinomas were detected.

Conclusions: Current follow-up of BNCs relies heavily on colposcopic assessment. A significant proportion of women with negative colposcopy was found to have high-grade histology in the first year of follow-up. We propose a more robust management algorithm to lower the probability of missed high-grade histology in this subgroup of women.

Glandular abnormalities of the cervix are rare and have always been a diagnostic challenge. Cytological screening seems to be less effective in glandular lesions than in squamous lesions (Krane et al, 2001). Furthermore, glandular lesions are often subtle, occurring at the edge of a more prominent visible squamous lesion. Owing to the endocervical location and the presence of skip lesions, glandular lesions are often missed during colposcopy. In addition, the mosaic pattern, punctation and leukoplakia, often associated with squamous lesions, are generally not seen with glandular lesions (Wright, 1999).

Changes in glandular cells where dyskaryosis cannot be excluded are grouped under borderline nuclear change in endocervical cells (BNCs; Evans et al, 1986). Typically, cell groups show either architectural or nuclear features suggesting cervical glandular intraepithelial neoplasia (CGIN). There may be crowded cells with pseudostratification with little nuclear abnormality or coarsely clumped chromatin with an entirely normal architecture. Borderline nuclear change in endocervical cells was recognised for the first time in the British Society of Clinical Cytology (BSCC) 1986 classification, and has subsequently been incorporated into the National Health Service Cervical Screening Programme (NHSCSP) management guidelines (Evans et al, 1986). In the Bethesda system the category of 'atypical glandular cells' (AGC) is broadly equivalent to BNCs, although this category is further subdivided according to: (i) the likely cell of origin and (ii) whether or not the cytopathologist 'favours neoplasia' (Solomon, 1990; Solomon et al, 2002; Cullimore and Waddell, 2010). 
Borderline nuclear change in endocervical cells is rare with a reported incidence of $0.04-1.8 \%$ in the literature (Meath et al, 2002; Daniel et al, 2005). The most commonly associated significant cervical pathology with BNCs is high-grade premalignant squamous abnormality. Frequently, benign conditions like endocervical polyps, cervicitis, cervical endometriosis, tubo endometriod metaplasia, and microglandular hyperplasia may be associated with BNCs (Schnatz et al, 2006). Moreover, it is not uncommon for BNCs to be associated with significant cervical lesions such as endocervical adenocarcinoma in situ (AIS) or adenocarcinoma of the cervix and endometrium.

Cytological diagnosis of BNCs is demanding for the cytologist and is not easily reproducible (Confortini et al, 2006). Correctly categorising the cell of origin and differentiating clinically significant changes from benign counterparts may be difficult (Simsir et al, 2003). Moreover, BNCs can be confused with borderline squamous abnormality, high-grade squamous abnormality and glandular involvement by squamous abnormality (Selvaggi, 1999).

Given the paucity of large prospective data and the absence of clear-cut algorithm or guideline, management of BNCs is not straightforward (Evans et al, 1986). Most of the data are based on AGC of The Bethesda System (2001; Solomon, 1990; Solomon et al, 2002). The American Society of Colposcopy and Cervical Pathology (ASCCP) guidelines recommend colposcopy with endocervical sampling for women with all subcategories of AGC (Levine et al, 2003). Endometrial sampling is recommended in women who are aged 35 years or over (Wright et al, 2002). Reflex HPV DNA or repeat cytology is considered unacceptable for the initial triage of AGC and AIS (Wright et al, 2002).

Since April 2012, high-risk human papillomavirus (HR HPV) triage has been incorporated into the NHSCSP (DOH, 2011). When borderline change in squamous or endocervical cells is reported on a cytology sample, a reflex HR HPV test is performed to assess the presence of HR HPV DNA and those who are positive for HR HPV are referred for colposcopy ((NHSCSP), 2010). Subsequent management is dependent on colposcopic and histological findings. However, women who are HR HPV negative are returned to routine recall; 3 years in the under 50s and 5 years in the over 50s. The NHSCSP publication (No 20) on colposcopy and programme management recommends colposcopic assessment, appropriate biopsy and selective use of endometrial sampling, in women with BNCs (Luesley and Leeson, 2010).

The HR HPV triage recommendations are based on the NHS Sentinel Sites HR HPV studies' data, that predominantly comprised of borderline squamous abnormalities (NHSCSP, 2011). Borderline squamous abnormality positive for $\mathrm{HR} \mathrm{HPV}$ is associated with cervical intraepithelial neoplasia (CIN 2) or worse histology, in 16-18\%, while limited evidence available shows that AGC positive for HR HPV is associated with high-grade histology (CIN 2/CIN 3/CGIN/AIS/invasive carcinoma) in 50-62\% (Kelly et al, 2011). Women with AGC, are reported to be at increased risk for clinically significant lesion during follow-up, despite a negative comprehensive evaluation (Chhieng et al, 2004; Schnatz et al, 2009). Furthermore, it is challenging to cytomorphologically distinguish BNCs from endocervical and endometrial origin. The reported rates of premalignant and malignant endometrial lesions in HR HPV-negative women with BNCs vary between 3 and 5\% (Saqi et al, 2006; Chen and Yang, 2008). Hence, the current follow-up seems questionable and illogical.

The objectives of our study were: (i) to review the clinical outcome of all women referred with BNCs, (ii) to determine the accuracy of colposcopy in identifying high-grade lesions in this subgroup and (iii) to propose a more robust management algorithm.

\section{MATERIALS AND METHODS}

Setting and population. This retrospective study was conducted at the Northumbria Healthcare Trust. The Northumbria Healthcare Trust, in the northeast of the UK, provides healthcare to over half a million people from North Tyneside to the Scottish borders and to Hexham and Haltwhistle, in the west of Northumberland. All smears from the region are processed in the regional cytology services of Royal Victoria Infirmary, in Newcastle upon Tyne. Colposcopy service is provided in four hospitals of the trust; Wansbeck Hospital, North Tyneside General Hospital, Hexham General Hospital and Blyth Community Hospital. Colposcopy services are provided by a group of five consultants, two colposcopy specialist nurses and colposcopy trainees under supervision.

Case selection. All smears coded as 'borderline smears' in the Excelicare Colposcopy database (AxSys, Paisley, UK; a database in use across the trust for colposcopy, containing all the patient details including referral details, ongoing clinic visits, diagnosis, treatment and discharge data, between January 2006 and December 2011), were manually searched to identify the term 'Borderline nuclear change with endocervical atypia'. This entity was not coded separately. The cytology department currently uses the 'Borderline nuclear change with endocervical atypia' category for women in whom the cytological changes are thought to be glandular in origin and are not sufficiently severe to be classed as dyskaryosis/glandular neoplasia.

All patients seen within 6 months of the index smear were included in the analysis. AxSys, Sunquest Integrated Clinical Environment (ICE) pathology requesting and reporting system and case records were reviewed to identify patient demographic characteristics, that is, age, parity, history of abnormal bleeding, smoking, use of hormonal contraception and hormone replacement therapy, previous smear and treatment history, colposcopic findings, histopathology results and subsequent cytology with follow-up details. All smears reported as endometrial atypia were excluded.

Women referred with BNCs underwent colposcopic assessment of the cervix with diagnostic biopsy of abnormal areas. Women with high-grade histology on diagnostic cervical biopsy were offered excisional biopsy. Women with complete excision of squamous lesions on large loop excision of transformation zone (LLETZ), were discharged with a view to perform repeat cervical smear in 6 months by the general practitioner, as per national guidelines (Luesley and Leeson, 2010). Women with low-grade histology on diagnostic punch biopsy were reviewed in 6 months in the colposcopy clinic for a repeat smear and colposcopy.

Women with satisfactory colposcopy (types 1 and 2 transformation zone) and negative colposcopy (no CIN or glandular abnormality with or without biopsy) were reviewed in 6 months for a cytological and colposcopic assessment. If these were normal, they were returned to routine recall (Luesley and Leeson, 2010). Endometrial biopsy and pelvic ultrasonography were offered to symptomatic women presenting with intermenstrual bleeding, postmenopausal bleeding, etc.

Histological diagnosis was based on high-grade histology (CIN 2/CIN 3/CGIN/invasive carcinoma) from a cervical punch biopsy, LLETZ biopsy, endometrial biopsy, hysterectomy (radical or total) or on cytology results (if the patient had cytological follow-up alone). If the subsequent histology during the first year of follow-up was high grade, this was included in the final diagnosis. Final histological diagnosis was based on concurrent or subsequent highest grade histology detected during the first year of follow-up.

Statistics. Sensitivity, specificity, positive predictive value (PPV), negative predictive value (NPV), pre-test and post-test probability 
of colposcopy, in identifying high-grade histology was determined (Akobeng, 2007).

\section{RESULTS}

From January 2006 to December 2011, 9001 new referrals were seen by colposcopy services at the Northumbria Healthcare Trust. Out of 3000 women with borderline smears identified from AxSys during the study period, 175 (2\%) were BNCs. Eight patients failed to attend colposcopy within 6 months of the index smear. Therefore, 167 (96\%) women, who were seen within 6 months of the index smear, formed the basis of the study.

Median age (range) of the study group was 43 years (20-68) and the median parity (range) was $2(0-5)$. Fifteen women $(9 \%)$ had previous treatment, while $35(21 \%)$ had previous abnormal smears. Twenty (12\%) women were symptomatic. Twenty-six (15\%) women were postmenopausal and one woman was pregnant. Forty-seven $(28 \%)$ were on some form of hormonal treatment (Supplementary Table 1).

Colposcopy was satisfactory in 156 women (93\%). One woman had a repeat cytology on her initial visit, while $11(7 \%)$ underwent cytology surveillance following a normal colposcopy (there were no obvious lesions to biopsy). One woman did not undergo colposcopy at the initial visit, as her biopsy showed inflammation following a colposcopy and diagnostic biopsy in a clinic abroad. Histology was available in 154 women (92\%); 9 (5\%) excision biopsies and 145 (87\%) diagnostic biopsies. Four women had excisional biopsies due to unsatisfactory colposcopy, two on suspicion of high-grade disease on colposcopy and one due to patient request. In two women, the reason for excisional biopsy was not documented. Thirty-seven women (22\%) were diagnosed with high-grade histology on initial assessment; $1(3 \%)$ on excision biopsy and 36 (97\%) on diagnostic biopsy (Figure 1).

Median follow-up (range) was 12 months (0-66). Twenty-seven (16\%) women had follow-up for 2 years as women with negative cytology and colposcopy were discharged to routine recall. In the first year, four high-grade histologies (7\%) were detected during follow-up of 55 women with low-grade histology on the initial assessment, while one high-grade histology (14\%), was detected in seven women with inadequate biopsy at the initial visit. Six highgrade histologies and one high-grade smear (severe dyskaryosis; $12 \%$ ) were detected in the first year follow-up of 60 women with satisfactory and negative colposcopy. One in seven women had previously undergone loop biopsy compared to 13 (25\%) in the remaining 53 women with negative colposcopy. Four of seven (57\%) women had a history of low-grade cytological abnormalities compared to $10(18 \%)$ in the remaining 53 women. The median age (range) of the seven women detected with high-grade histology on follow-up was 48 (30-62) years. One adenocarcinoma of the cervix was detected on follow-up of 10 women with unsatisfactory colposcopy (Figure 1).

Overall, 50 (30\%) high-grade histologies including two invasive carcinomas of the cervix were detected: 37 (74\%) during initial assessment and 13 (26) in the subsequent year of follow-up. Thirty-six (72\%) were squamous lesions, while 12 (24\%) were pure glandular lesions. Fifty-one (30\%) women had low-grade histology and $30(18 \%)$ had benign lesions.

The prevalence of high-grade histology in women $<35$ years of age was 19 out of $39(49 \%)$ and this was 31 out of $128(24 \%)$ in the over 35s. Median age of women with pure glandular abnormality was 34 years. Among the 12 women with pure glandular lesions, 10 had satisfactory colposcopy. None were suspected to have glandular or high-grade lesions on colposcopy, four were suspected to have benign lesions, while six were thought to have low-grade lesions (data not shown).
Only 11 of the 48 women with satisfactory colposcopy and highgrade histology were thought to be high grade on colposcopy. The sensitivity, specificity, PPV and NPV of colposcopy in detecting high-grade lesions were $23,94,61$ and $73 \%$, respectively. The pretest probability of colposcopy for high-grade histology was $31 \%$, while post-test probability was $61 \%$. The pre-test probability of colposcopy for glandular abnormality was $8 \%$, while the post-test probability was $11 \%$ (Table 1 ).

Both women diagnosed with invasive carcinomas had a normal appearance on visual and colposcopic examination. One of them was a 43-year-old woman referred with a borderline glandular abnormality and was suspected to have a benign lesion on colposcopy. Cervical punch biopsy-confirmed CIN 3 and subsequent LLETZ diagnosed a FIGO stage 1A1 squamous cell carcinoma, which was completely excised (Pecorelli, 2009). Her subsequent cytology had been negative until her last follow-up.

The other woman was a 64-year-old postmenopausal, heavy smoker, referred with a borderline glandular smear. She had an unsatisfactory colposcopy and benign histology on punch biopsy. A follow-up cytology after 6 months was suspicious for glandular neoplasia. A LLETZ biopsy confirmed an invasive adenocarcinoma FIGO stage 1B1, which was incompletely excised (Pecorelli, 2009). She subsequently underwent radical hysterectomy with bilateral salpingo-oophorectomy and bilateral pelvic lymph node dissection. However, two and a half years later, she died from aspiration pneumonia with radiologically suspected lung metastasis.

\section{DISCUSSION}

This is the largest series of women with BNCs reported on liquidbased cytology from the UK based on the BSCC classification. The rate of high-grade histology in our series was $30 \%$. Twenty-four percent of these were pure glandular lesions. These figures are similar to the rates reported in the literature; $20-50 \%$ for $\geqslant C I N 2$ and $12-46 \%$ for pure glandular lesions (Mohammed et al, 2000; Geier et al, 2001; Scheiden et al, 2004; Schnatz et al, 2006).

The follow-up study from Sentinel Sites noted that, in the HR HPV-positive women with low-grade cytological abnormalities found to have satisfactory and negative colposcopy, the risk of CIN 2 or worse was $4.4 \%$, at 3 years (Kelly et al, 2012). Based on the above reassuring figures, currently in the UK, if HR HPV-positive women with BNCs have a negative satisfactory colposcopy, they are returned to routine recall. However, HR HPV-negative women with BNCs do not have a colposcopic assessment at all. This guidance was based on only 17 women with BNCs: $0.5 \%$ of all the women with low-grade cytological abnormalities in the NHS Sentinel Sites study (Luesley and Leeson, 2010; NHSCSP, 2011). The rate of HR HPV positivity in women with BNCs was $71 \%$, while that of high-grade histology (CIN 2 or worse) was $33 \%$ (NHSCSP, 2011). In addition, current follow-up relies heavily on colposcopic assessment and impression. Normal colposcopic examination and cervical biopsy may not necessarily be reassuring in glandular abnormalities, as the lesion may be small and may be located higher up in the endocervical canal. Many cases of biopsyconfirmed AIS have no observed colposcopic abnormalities and even combination of cytological testing and colposcopy can miss small endocervical adenocarcinoma or AIS localised in the endocervical canal (Luesley et al, 1987; Ullal et al, 2009; Talaat et al, 2012). Accuracy of colposcopy ranges from 9 to $50 \%$ in recognising biopsy-confirmed glandular abnormalities (Ullal et al, 2009; Talaat et al, 2012). Furthermore, the accuracy of colposcopy in predicting high-grade disease, is lower in low-grade cytological abnormality (12\%) compared to high-grade cytological abnormality (58\%; Pretorius et al, 2001). 

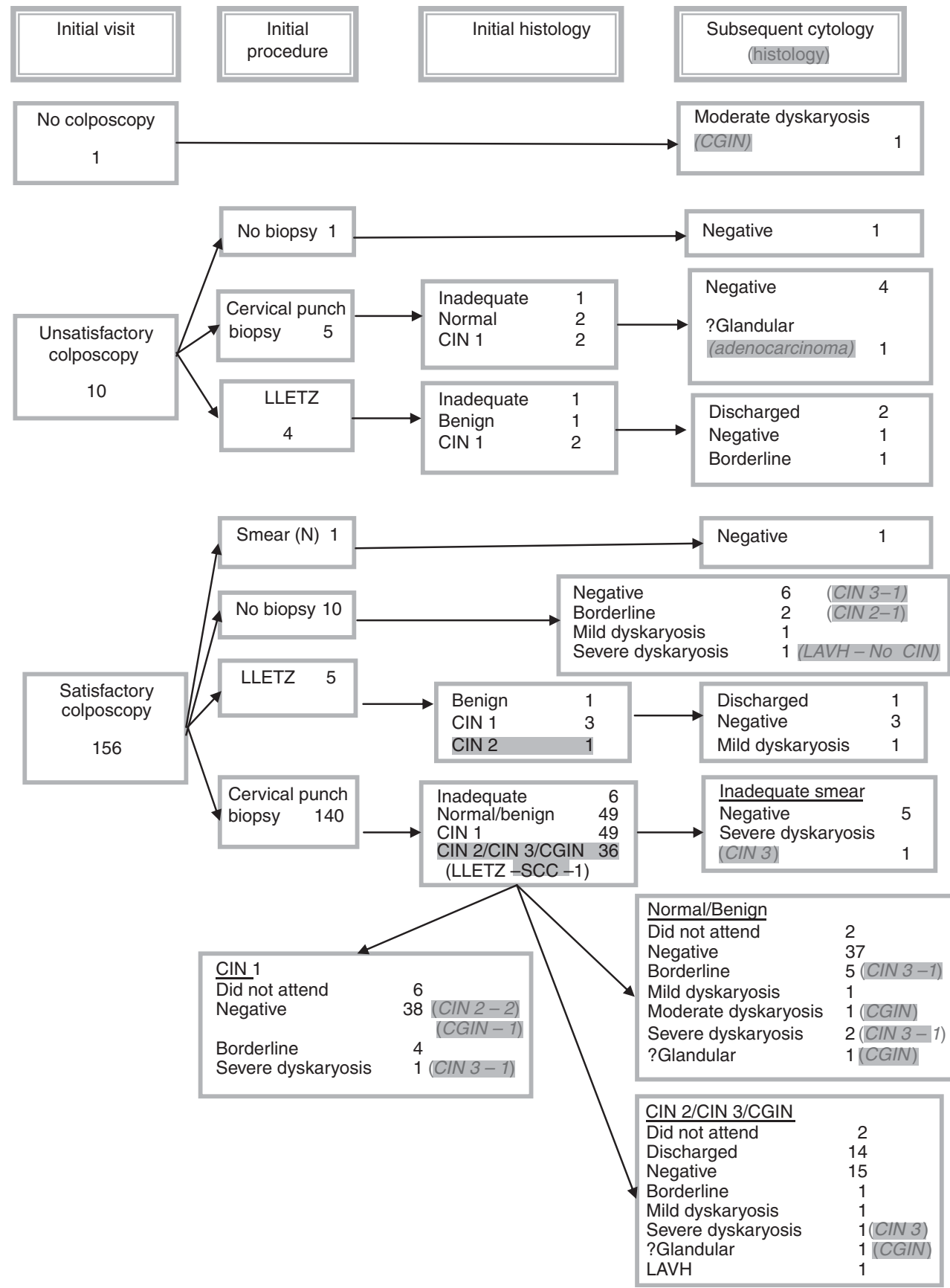

Figure 1. Initial management and outcome of subsequent follow-up. Abbreviations: CGIN = cervical glandular intraepithelial neoplasia; $\mathrm{CIN}=$ cervical intraepithelial neoplasia; LAVH = laparoscopy-assisted vaginal hysterectomy; LLETZ=large loop excision of transformation zone; $\mathrm{SCC}=$ squamous cell carcinoma.

In our study, none of the pure glandular lesions were suspected to be glandular or high-grade disease on colposcopy, in spite of being examined by the British Society of Colposcopy and Cervical Pathology (BSCCP) accredited colposcopists. Colposcopic post-test probability in glandular lesions was not different from pre-test probability ( $8 v s 11 \%)$. This reiterates the difficulty in identifying glandular lesions by colposcopy. Formulating a follow-up management plan based on colposcopy for an abnormal smear significantly associated with glandular abnormality, is clearly unreliable.

Delayed diagnosis of significant pathology during follow-up of BNCs is variable (1-11\%) in the literature (Smith et al, 2006; Schnatz et al, 2009; Patel et al, 2010; Valdini et al, 2013). In our study, 6 (10\%) high-grade histology and one high-grade smear (transferred care for excisional biopsy at another trust) were detected on follow-up within 1 year of the initial colposcopic visit in 60 women known to have BNCs with negative colposcopy. This is much higher than the reported rates in the Sentinel Sites (NHSCSP, 2011). Such a high rate of high-grade histology during follow-up, possibly reflecting originally unidentified or missed lesions, is indeed very concerning.

Should we then follow an aggressive approach with universal excisional biopsy in all women with BNCs due to inaccuracy of colposcopy in identifying glandular lesions? Such a policy in women over 35 years of age was suggested by a recent study from the UK (Patel et al, 2010). However, such a policy could lead to overtreatment, with its surgical and reproductive morbidities, especially in young women. Moreover, BNCs in women over 
35 years are more likely to be endometrial in origin, that is, hyperplasias and carcinomas (DeSimone et al, 2006). In our study, $24 \%$ of over 35 s compared to $49 \%$ of under 35 s had high-grade histology. We would therefore disagree with such an approach.

The rates of endometrial adenocarcinoma in women with AGC, reported in the literature, vary between 0.2 and 29\% (Scheiden et al, 2004; Zhao et al, 2009). Rates reported in the UK vary between 1 and 2\% (Mohammed et al, 2000). Three to five percent endometrial lesions (hyperplasia and adenocarcinoma) have been reported in HR HPV-negative women with AGC (Saqi et al, 2006; Chen and Yang, 2008). There is a wide variation in practice regarding endometrial evaluation of women with BNCs in the UK, as there is no definitive recommendation from the BSCCP. A recent study proposed that women over 35 years should undergo endometrial sampling only if they are symptomatic or if colposcopy is satisfactory and normal (Patel et al, 2010). Furthermore, HR HPV is not a risk factor for endometrial malignancy. Hence, attempting to triage a cellular abnormality, which is not easy to interpret and not easily reproducible (Simsir et al, 2003) in women who are also at risk of endometrial carcinoma, based on HR HPV may inevitably lead to missed diagnoses. No endometrial adenocarcinomas were detected in our series. This could be due to under evaluation of endometrial pathology.

The ASCCP guidelines recommend referral of all women with AGC to immediate colposcopy. Colposcopy, endocervical curettage and/or endometrial biopsy are performed. High-risk human papillomavirus testing is performed as part of primary evaluation. If the post-colposcopy evaluation does not reveal any CIN or glandular abnormalities, cytology and HPV testing is repeated at 6 months in HR HPV-positive women or at 12 months in HR HPV-negative women. Those with negative evaluation (no CIN or glandular abnormalities) with 'AGC-favour neoplasia' are subjected to diagnostic excisional biopsy (Wright et al, 2002; Wright et al, 2007).

We propose a rational and reliable management pathway for patients with BNCs. We suggest that all HR HPV-positive women with BNCs should undergo colposcopy \pm biopsy. Multidisciplinary team discussion with cytologists and pathologists in a colposcopy correlation meeting should be considered in women with negative

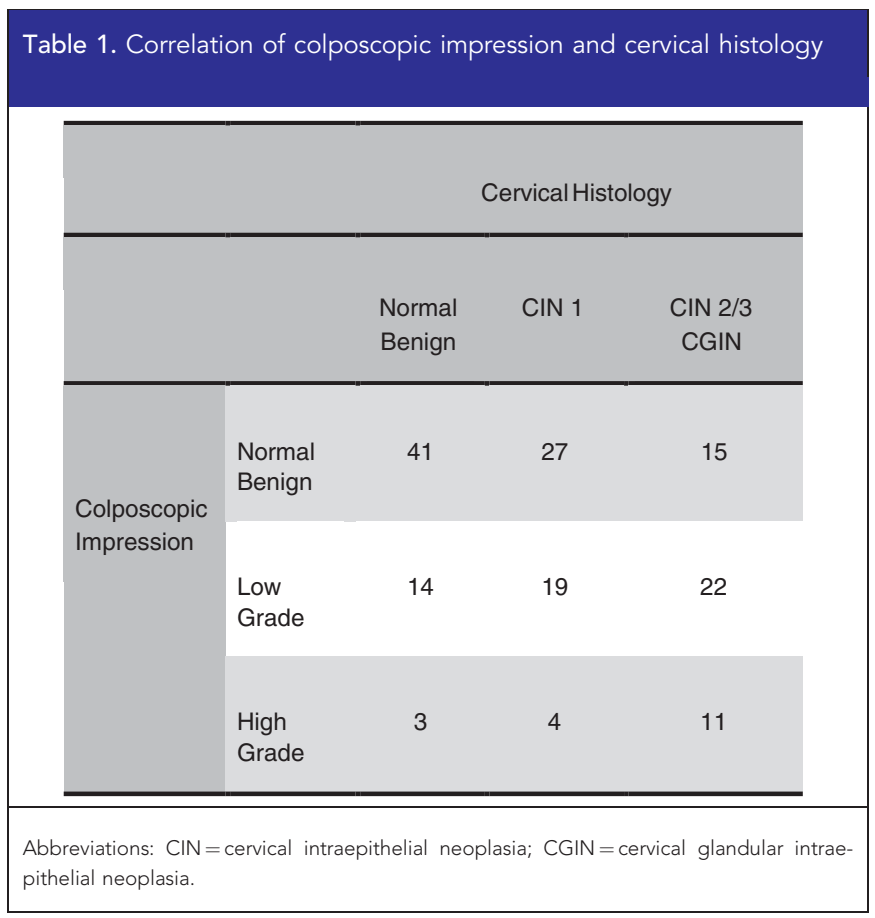

colposcopy before formulating an individualised management plan. Women with negative colposcopy should have a repeat smear including endocervical sampling as well as an HR HPV test in 6 months time. In addition, women who are HR HPV negative, over 40 years of age, high risk (in view of chronic anovulation or morbid obesity), or symptomatic, should be referred to an appropriate specialist for a pelvic ultrasound and endometrial biopsy, to exclude an endometrial pathology (Figure 2).

Approximately, 3.7 million smears are performed every year in the UK. In total, $18500(0.5 \%)$ of all smears are likely to be BNCs, and if $70 \%$ of them are HR HPV positive, 12950 women with BNCs are likely to be seen with colposcopy every year in the UK (Kelly et al, 2011). Over 50\% of such referrals are likely to result in a negative colposcopic examination (Kelly et al, 2012). Assuming a risk of $10 \%$ missed diagnoses, there is a risk that $\sim 650$ women with high-grade lesions are being discharged to routine recall. With HR HPV triage, the time to diagnosis of missed lesions could be 3-5 years depending on age. Our suggested algorithm would potentially diagnose these lesions at the 6-month follow-up (Supplementary Table 2).

The results of our study should be interpreted with caution. This was a retrospective review and our study population was identified by a manual search of all borderline smears in the trust colposcopy database, thereby raising the possibility of missing data. Furthermore, missing long-term follow-up data due to failure to attend or due to discharge to the community might not have represented the true follow-up picture. In addition, this study preceded the HR HPV triage introduction. There being no separate guidelines on the management of BNCs in the UK, and due to its rarity, women with BNCs were managed identically to women with borderline nuclear change in squamous cells. Lack of guidance from BSCCP led to variation in the extent of evaluation and management of BNCs by individual accredited colposcopists. Consequently, the results might not represent the true extent of the pathology due to

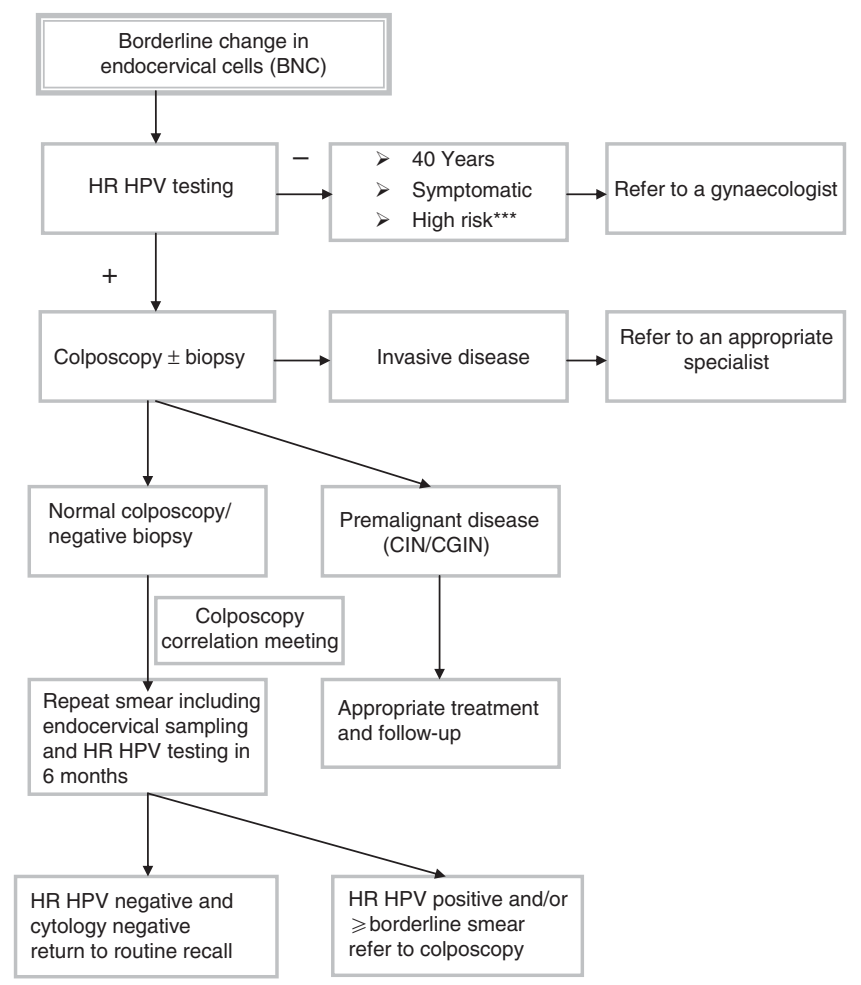

Figure 2. Algorithm for managing women with $\mathrm{BNCs}^{\star \star \star}$. Abbreviations: $\mathrm{CGIN}=$ cervical glandular intraepithelial neoplasia; $\mathrm{CIN}=$ cervical intraepithelial neoplasia; HR HPV = high-risk human papillomavirus. ${ }^{\star \star \star}$ Chronic anovulation, morbid obesity (Zhao et al, 2009). 
inadequate evaluation. However, this might reflect the current practice and the true picture in most hospitals offering a colposcopy service in the UK.

Literature review on HR HPV triage and AGC shows that the HR HPV prevalence rates vary between 20 and 71\%, while the PPV for high-grade lesions ranges from 50 to $62 \%$. This is much higher than for borderline nuclear change in squamous cells (16-17\%; Derchain et al, 2004; Krane et al, 2004; Irvin et al, 2005; Saqi et al, 2006; Chen and Yang, 2008; Sharpless et al, 2009). Such a high association with significant disease in the HR HPV-positive subgroup, if proven in large prospective studies in future, might mandate a more aggressive management approach, possibly a policy of excisional biopsy in all HR HPV-positive women with BNCs.

\section{CONCLUSION}

Borderline nuclear change in endocervical cells is a rare cytological abnormality; however, it is associated with a significant risk of high-grade histology, including glandular lesions and invasive carcinomas. There is an increased risk for clinically significant disease during follow-up, in spite of an initial negative comprehensive evaluation. Current HR HPV triage follow-up of BNCs relies heavily on colposcopic assessment, which has limitations in the evaluation of glandular lesions. In our study, a significant proportion of women with negative colposcopy were found to have high-grade histology within the first year of follow-up. We have proposed a more robust and comprehensive management algorithm to lower the probability of undiagnosed or missed high-grade histology in this subgroup of women. This suggested algorithm requires validation through well-designed prospective studies.

\section{REFERENCES}

Akobeng AK (2007) Understanding diagnostic tests 2: likelihood ratios, pre- and post-test probabilities and their use in clinical practice. Acta Paediatr 96(4): 487-491.

Chen L, Yang B (2008) Assessment of reflex human papillomavirus DNA testing in patients with atypical endocervical cells on cervical cytology. Cancer 114(4): 236-241.

Chhieng DC, Gallaspy S, Yang H, Roberson J, Eltoum I (2004) Women with atypical glandular cells: a long-term follow-up study in a high-risk population. Am J Clin Pathol 122(4): 575-579.

Confortini M, Di Bonito L, Carozzi F, Ghiringhello B, Montanari G, Parisio F, Prandi S. Cytology GIWGfC (2006) Interlaboratory reproducibility of atypical glandular cells of undetermined significance: a national survey. Cytopathology 17(6): 353-360.

Cullimore JE, Waddell C (2010) Cervical cytology and glandular neoplasia. BJOG 117(9): 1047-1050.

Daniel A, Barreth D, Schepansky A, Johnson G, Capstick V, Faught W (2005) Histologic and clinical significance of atypical glandular cells on pap smears. Int J Gynaecol Obstet 91(3): 238-242.

Derchain SF, Rabelo-Santos SH, Sarian LO, Zeferino LC, de Oliveira Zambeli ER, do Amaral Westin MC, de Angelo Andrade LA, Syrjanen KJ (2004) Human papillomavirus DNA detection and histological findings in women referred for atypical glandular cells or adenocarcinoma in situ in their Pap smears. Gynecol Oncol 95(3): 618-623.

DeSimone CP, Day ME, Tovar MM, Dietrich 3rd CS, Eastham ML, Modesitt SC (2006) Rate of pathology from atypical glandular cell Pap tests classified by the Bethesda 2001 nomenclature. Obstet Gynecol 107(6): $1285-1291$.

DOH (2011) NHS Cervical Screening Programme: implementing HPV triage for women with mild or borderline cervical screening test results and HPV test of cure, Programmes DoHNCS (ed).

Evans DM, Hudson EA, Brown CL, Boddington MM, Hughes HE, Mackenzie EF, Marshall T (1986) Terminology in gynaecological cytopathology: report of the Working Party of the British Society for Clinical Cytology. J Clin Pathol 39(9): 933-944.

Geier CS, Wilson M, Creasman W (2001) Clinical evaluation of atypical glandular cells of undetermined significance. Am J Obstet Gynecol 184(2): 64-69.

Irvin W, Evans SR, Andersen W, Jazaeri A, Taylor P, Stoler M, Pastore L, Rice L (2005) The utility of HPV DNA triage in the management of cytological AGC. Am J Obstet Gynecol 193(2): 559-565, discussion 565-7.

Kelly RS, Patnick J, Kitchener HC, Moss SM. Group NHSI (2011) HPV testing as a triage for borderline or mild dyskaryosis on cervical cytology: results from the Sentinel Sites study. Br J Cancer 105(7): 983-988.

Kelly RS, Walker P, Kitchener H, Moss SM (2012) Incidence of cervical intraepithelial neoplasia grade 2 or worse in colposcopy-negative/human papillomavirus-positive women with low-grade cytological abnormalities. BJOG 119(1): 20-25.

Krane JF, Granter SR, Trask CE, Hogan CL, Lee KR (2001) Papanicolaou smear sensitivity for the detection of adenocarcinoma of the cervix: a study of 49 cases. Cancer 93(1): 8-15.

Krane JF, Lee KR, Sun D, Yuan L, Crum CP (2004) Atypical glandular cells of undetermined significance. Outcome predictions based on human papillomavirus testing. Am J Clin Pathol 121(1): 87-92.

Levine L, Lucci 3rd JA, Dinh TV (2003) Atypical glandular cells: new Bethesda Terminology and Management Guidelines. Obstet Gynecol Surv 58(6): 399-406.

Luesley D, Leeson S (2010) Colposcopy and Programme Management: Guidelines for the NHS Cervical Screening Programme. 2nd edn. NHS Cancer Screening Programme: Sheffield.

Luesley DM, Jordan JA, Woodman CB, Watson N, Williams DR, Waddell C (1987) A retrospective review of adenocarcinoma-in-situ and glandular atypia of the uterine cervix. Br J Obstet Gynaecol 94(7): 699-703.

Meath AJ, Carley ME, Wilson TO (2002) Atypical glandular cells of undetermined significance. Review of final histologic diagnoses. J Reprod Med 47(4): 249-252.

Mohammed DK, Lavie O, de B Lopes A, Cross P, Monaghan JM (2000) A clinical review of borderline glandular cells on cervical cytology. BJOG 107(5): 605-609.

(NHSCSP) (2010) NHSCSP. HPV Triage and Test of Cure Protocol for Women Aged 25 to 64 Years.

NHSCSP (2011) Evaluation of the Sentinel Sites for HPV triage and test of cure. Report to the NHS Cancer Screening Programmes.

Patel A, Thampy N, Hemming D, Naik R (2010) A clinical review of borderline glandular cells reported on liquid-based cervical cytology. BJOG 117(9): 1051-1059.

Pecorelli S (2009) Revised FIGO staging for carcinoma of the vulva, cervix, and endometrium. Int J Gynaecol Obstet 105(2): 103-104.

Pretorius RG, Belinson JL, Zhang WH, Burchette RJ, Elson P, Qiao YL (2001) The colposcopic impression. Is it influenced by the colposcopist's knowledge of the findings on the referral Papanicolaou smear? J Reprod Med 46(8): 724-728.

Saqi A, Gupta PK, Erroll M, Babiac A, Blackmun D, Mansukhani M, Vazquez M (2006) High-risk human papillomavirus DNA testing: a marker for atypical glandular cells. Diagn Cytopathol 34(3): 235-239.

Scheiden R, Wagener C, Knolle U, Dippel W, Capesius C (2004) Atypical glandular cells in conventional cervical smears: incidence and follow-up. BMC Cancer 4: 37.

Schnatz PF, Guile M, O'Sullivan DM, Sorosky JI (2006) Clinical significance of atypical glandular cells on cervical cytology. Obstet Gynecol 107(3): 701-708.

Schnatz PF, Surette A-M, O'Sullivan DM (2009) Delayed diagnoses after atypical glandular cells on cervical cytology. J Low Genit Tract Dis 13(4): 237-243.

Selvaggi SM (1999) Please! No "AGUS" swamp. Atypical glandular cells of undetermined significance. Diagn Cytopathol 21(3): 157-158.

Sharpless KE, O'Sullivan DM, Schnatz PF (2009) The utility of human papillomavirus testing in the management of atypical glandular cells on cytology. J Low Genit Tract Dis 13(2): 72-78.

Simsir A, Hwang S, Cangiarella J, Elgert P, Levine P, Sheffield MV Roberson J, Talley L, Chhieng DC (2003) Glandular cell atypia on Papanicolaou smears: interobserver variability in the diagnosis and prediction of cell of origin. Cancer 99(6): 323-330. 
Smith MC, Keech SE, Perryman K, Soutter WP (2006) A long-term study of women with normal colposcopy after referral with low-grade cytological abnormalities. BJOG 113(11): 1321-1328.

Solomon D (1990) The 1988 Bethesda System for reporting cervical/vaginal cytologic diagnoses: developed and approved at the National Cancer Institute Workshop in Bethesda, Maryland, December 12-13, 1988. Hum Pathol 21(7): 704-708.

Solomon D, Davey D, Kurman R, Moriarty A, O'Connor D, Prey M, Raab S, Sherman M, Wilbur D, Wright Jr T, Young N. Forum Group MBethesda W (2002) The 2001 Bethesda System: terminology for reporting results of cervical cytology. JAMA 287(16): 2114-2119.

Talaat A, Brinkmann D, Dhundee J, Hana Y, Bevan J, Irvine R, Bailey S, Woolas R (2012) Risk of significant gynaecological pathology in women with glandular neoplasia on cervical cytology. Cytopathology 23(6): 371-377.

Ullal A, Roberts M, Bulmer JN, Mathers ME, Wadehra V (2009) The role of cervical cytology and colposcopy in detecting cervical glandular neoplasia. Cytopathology 20(6): 359-366.

Valdini AF, Augart CL, Olivieri M (2013) Delayed diagnoses of cervical intraepithelial neoplasia and cancer after negative evaluation for atypical glandular cell pap smear: does age matter? J Low Genit Tract Dis 17(4): 390-396.
Wright Jr TC, Cox JT, Massad LS, Twiggs LB, Wilkinson EJ. Conference AS-SC (2002) 2001 consensus guidelines for the management of women with cervical cytological abnormalities. JAMA 287(16): 2120-2129.

Wright Jr TC, Massad LS, Dunton CJ, Spitzer M, Wilkinson EJ, Solomon D. Conference AS-SC (2007) 2006 consensus guidelines for the management of women with abnormal cervical screening tests. J Low Genit Tract Dis 11(4): 201-222.

Wright VC (1999) Colposcopy of adenocarcinoma in situ and adenocarcinoma of the uterine cervix: differentiation from other cervical lesions. J Low Genit Tract Dis 3(2): 83-97.

Zhao C, Florea A, Onisko A, Austin RM (2009) Histologic follow-up results in 662 patients with Pap test findings of atypical glandular cells: results from a large academic womens hospital laboratory employing sensitive screening methods. Gynecol Oncol 114(3): 383-389.

This work is published under the standard license to publish agreement. After 12 months the work will become freely available and the license terms will switch to a Creative Commons AttributionNonCommercial-Share Alike 3.0 Unported License.

Supplementary Information accompanies this paper on British Journal of Cancer website (http://www.nature.com/bjc) 